\title{
Proceed to the Diagnosis of an Annular Lesion
}

\section{(1) Somnath Das, (1) Aniruddha Mandal, (1) Pranjal Parveen, (1) Subhadeep Mallick, (1) Olympia Rudra, (1) Pramit Nandy, (1) Aishwarya Prakash, (1) Subhasmita Baisya, (1) Pratik Dey, (1) Sudip Mandal}

Institute of Post Graduate Medical Training and Reasearch, Clinic of Dermatology, Kolkata, India

Keywords: Annular lesion, Lichen planus, Hypertrophic lichen planus

\section{Dear Editor,}

Lichen planus (LP), the prototype of lichen dermatoses is an idiopathic inflammatory disease of the skin and mucous membrane. Classic LP presents with characteristic 5Ps- polygonal, pruritic, plane (flat-topped), purple (violaceous) papules and plaques that favours the extremities [1,2]. Prevalence of LP is $1 \%$ in general population. Nearly two-thirds of cases of LP presents between the ages of 50 and 60 years with peak onset between 55 and 74 years [3,4].

LP has a lot of variants including oral, nail, linear, annular, atrophic, hypertrophic, inverse, eruptive, bullous, ulcerative, LP pigmentosus, lichen planopilaris, vulvovaginal, actinic, LP pemphigoides and LPlupus erythematosus overlap [5].

Annular LP occurs when papules spread peripherally and central area resolves. The annular edge is slightly raised and cental area is hyperpigmented or skin coloured. Annular lesion occurs in 10\% patients with LP and are usually scattered among classical typical lesions [6].

A 32 year old female presented to our dermatology OPD with extremely itchy, hyperpigmented keratotic plaques on trunk and extremities (Figure 1). The lesions were present for last 8 months. She had taken ayurvedic medicines for the same with no relief. The lesions initially started as small, itchy papules on wrists, arms, ankles and legs; and gradually increased in size with centrifugal expansion and similar lesions also appeared on trunk. Some of the lesions cleared in the centre to assume annular configruation.

On examination, there were hyperpigmented, keratotic plaques with well-defined border on the extensor aspects of wrists, forearm, legs, and trunk with some lesions on dorsal foot and wrists having annular configuration with normal skin in the centre. The lesions were of $1-3 \mathrm{~cm}$ in size. On application of mineral oil and using a magnifying lens, Wickham striae was demonstrated. There were no lesions on oral and genital mucosa. Nails and hair were normal.

We considered differentials of annular and hypertrophic LP, prurigo nodularis and lupus vulgaris.

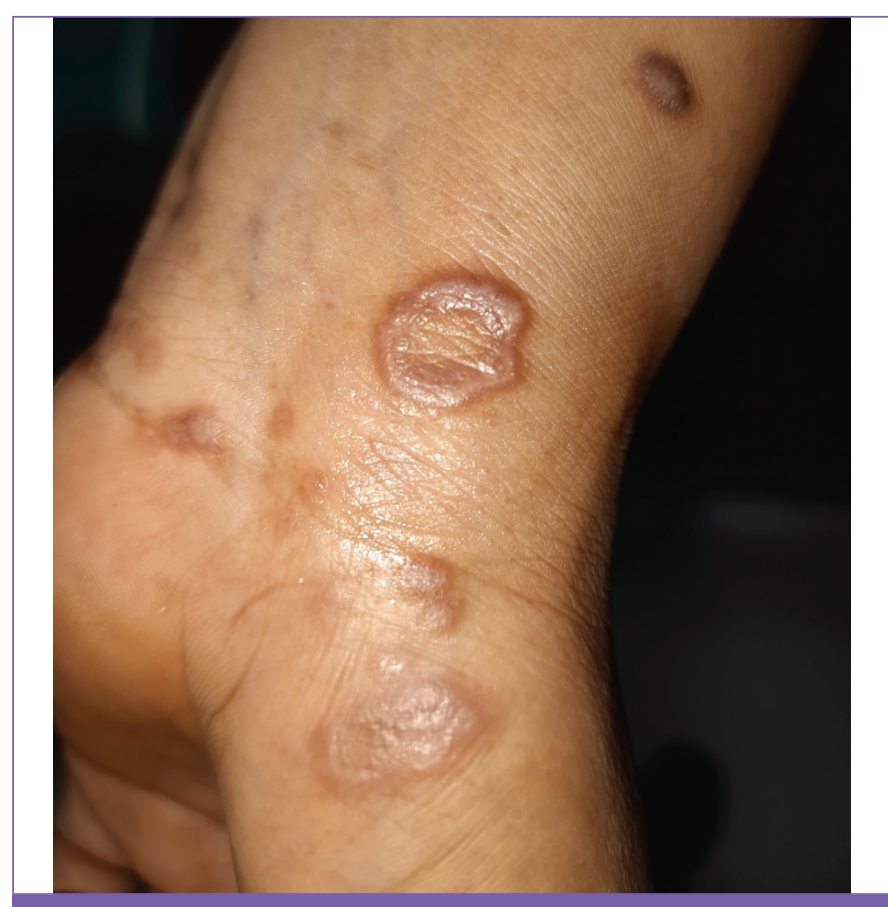

Figure 1. Keratotic plaques on trunk and extremities 


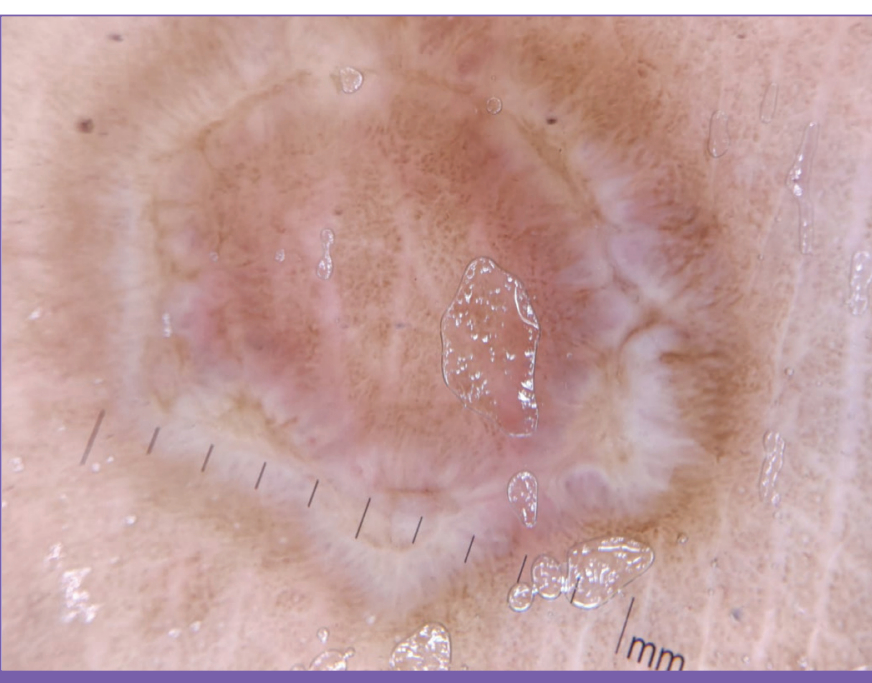

Figure 2. Dermoscopic picture

Dermoscopy of the lesions revealed white, broad reticular striae on purplish to brownish background. Fine red dots around the striae were also noted (Figure 2).

Laboratory investigations showed normal blood counts, fasting hyperglycemia with elevated serum cholesterol level. Serological tests for human immunodeficiency virus-1 and 2, Hep B and Hep C were negative. Mantoux test showed no erythema and induration. Chest X-ray was within normal limits.

Biopsy was taken from one such annular and histopathological examination using haematoxylin and eosin stain was done. It revealed lymphocytic infiltrate in a diffuse band like pattern in the upper dermis along with basal cell vacuolation with subepidermal clefts (Figure 3).

She was treated with oral prednisolone $30 \mathrm{mg}$ daily, proton pump inhibitors, anti-histaminics and topical Emollient lotion for 4 weeks following which smaller lesions resolved and large lesions reduced in size and thickness.

Based on the above findings, a diagnosis of hypertrophic LP and Annular LP was reached.

Pure annular LP is rare. In most cases of LP, $10 \%$ of lesions are annular. Another rare variant is annular atrophic LP. Annular lesions are usually associated with oral and genital lesions which were absent in our case.

\section{Ethics}

Informed Consent: Consent form was filled out by all participants.

Peer-review: Internally peer-reviewed.

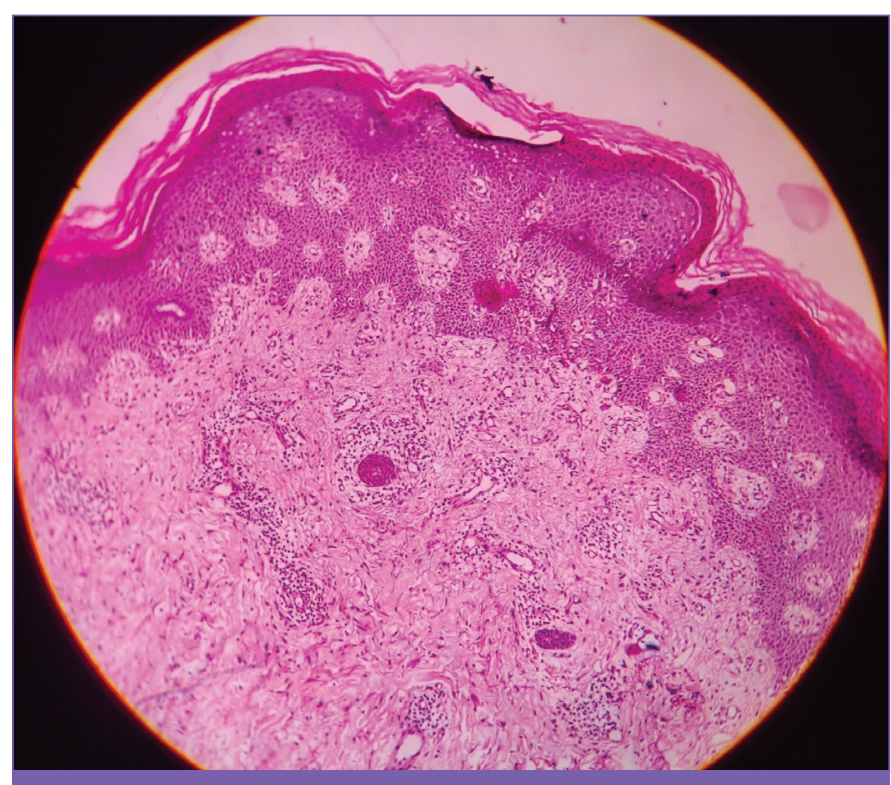

Figure 3. Histopathological picture: Iymphocytic infiltrate in a diffuse band like pattern in the upper dermis along with basal cell vacuolation with subepidermal clefts

\section{Authorship Contributions}

Surgical and Medical Practices: S.D., O.R., P.N., A.P., S.Man., Concept: S.D., A.M., P.P., S.M., S.B., S.Man., Design: S.D., P.P., P.N., S.M., Data Collection or Processing: S.D., P.N., P.D., Analysis or Interpretation: S.M., A.P., Literature Search: S.D., P.P., O.R., A.P., P.D., Writing: S.D., P.P., O.R., P.N., A.P., P.D., S.Man.

Conflict of Interest: No conflict of interest was declared by the authors.

Financial Disclosure: The authors declared that this study received no financial support.

\section{References}

1. Boyd AS, Neldner KH. Lichen planus. J Am Acad Dermatol. 1991;25:593-619.

2. Wickham L. Sur un signe pathogomonique du lichen du Wilson (lichen plan) Ann Dermatol Syphiligr 1895;6:517-520.

3. Mangold AR, Pittelkow MR. Lichenoid and Granulomatous Disorders In:Sewon Kang, editor. Fitzpatrick's Dermatology. 9th edn. United States: McGraw-Hill; 2019. p. 529.

4. Sripathi H, Kudur MH, Prabhu S, Pai SB. Punctate keratotic papules and plaques over palm. Diagnosis: Hypertrophic lichen planus of palm. Indian J Dermatol Venereol Leprol 2010;76:449.

5. Shiohara T, Mizukawa Y. Lichen Planus and Lichenoid Dermatoses. In: Bolognia JL, Schaffer VJ, Cerroni L. Dermatology, 4th ed. China: Elsevier; 2018. p. 236

6. Eyler JT, Garib G, Thompson KR, Dahiya M, Swan JW. Annular atrophic lichen planus responds to hydroxychloroquine and acitretin. Cutis 2017;100:119122. 